\title{
Design and Realization of Simulation System Combined With Virtual Reality of Tool Controller Mechanism for Machining Center Based on Irai Software
}

\author{
Meng-Ying LIU ${ }^{1 a}$, Wei ZHAO ${ }^{1 b}$, Kuan WANG ${ }^{1 c}$, Yong-Gang CHEN12d and \\ Chen-Yang WEI ${ }^{2 e}$
}

${ }^{1}$ Tianjin Key Laboratory of High Speed Cutting \&Precision Machining (TUTE), Tianjin, China

${ }^{2}$ Beij geruina LTD., Beijing, China

a358413334@qq.com, bzhaowei@tju.edu.cn, c86340166@qq.com dskyline_wei@163.com, e13910760339@139.com

Keywords: Thus the simulation system, Machining center tool change, PLC, Irai, Mechanical and electrical integration.

\begin{abstract}
In order to solve the problem that the training equipment is few and the training items are single, the students can not connect the learned skills with the actual production of the enterprise ,a new idea is put forward to control the virtual 3D-model with the electronic components such as PLC, relay, switch and so on in Irai software. Through establishing contact between PLC and the model of tool controller mechanism for machining center based on Irai software, a platform is built for the experiments and training of mechatronics such as machine control, PLC and CNC machine tools installation and maintenance. The mechanical structure with large space, high cost and complicated structure can be virtual, and the electrical control part is real, thus solving the problems in the actual teaching and promoting the development of mechatronics teaching.

Nowadays, virtual reality technology (referred to as VR) is gradually integrated into all aspects of our lives.VR is a kind of technology which provide immersion feeling in interactive threedimensional environment that generates on the computer by using computer graphics system, various realities and interface devices. In the school teaching level,VR technology can solve the lack of experimental equipment and security risks in physical experimental equipment in teaching of various schools.For example, in the current PLC simulation training courses, students can only program on the PLC to achieve controlling motor rotation and LED lights[1].Large, and complex mechanical structure can not be truly controlled.

Engineering Training Center of Tianjin University of Technology and Education introduced a number of vertical machining centers, which had a good effect in training teaching.In order to make students having a more in-depth understanding of the NC system automation and make the further development of the mechatronics teaching,a new idea of real - time PLC control training for 3D virtual mechanical mechanism by using VR technology is presented.By generating solid modeling in Catia and other 3D drawing software and communicating with the external real PLC through the virtual control platform building by Irai,the real control on the virtual three-dimensional model will be realized.With the simulation system combined with virtual reality applying in the mechatronics training,the problems of laboratory teaching will be solved.
\end{abstract}

\section{Introduction of Iria Software}

Irai is a software for three-dimensional modeling and simulation.By external 3D modeling software, it can quickly create a 3D interactive simulation model of the automation system. With Irai simulation software, designers of industrial equipment and automation systems are able to test their products in realistic and interactive 3D virtual environments and monitor the behavior of analog devices at any time.By connecting a 3D simulation model with an external controller such as a PLC or an embedded virtual controller, Irai is able to reproduce the real-world behavior of a device or machine in a fully virtualized environment[2].Specific process to use shows in Figure 1 below. 


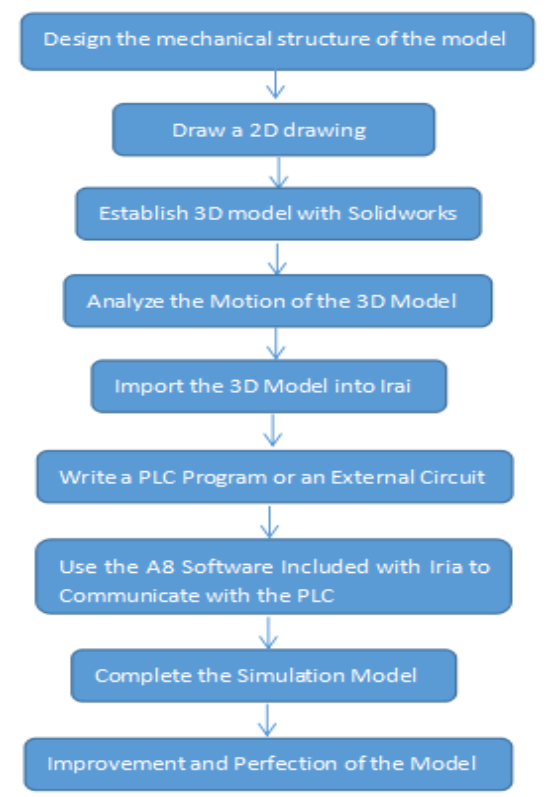

Figure 1. Irai Software Using Flowcharts

Irai 's main software is Virtual Universe Pro V2.This software is mainly to take simulation on the specific movement of the 3D-model and verify the correctness of the design of the 3D-model in the environment and platform provided by PC after the 3D-model built by Solidworks and other 3Dmodeling software is imported[3]. Thus the potential security risks due to the uncertainy of machine motion in the actual experiment are avoided.And the authenticity of the software can make the operator feeling immersive and get very good results.

Irai comes with the communication software A8.It can combine external real PLC and electrical lines with the 3D model in virtual platform provided by PC and perform motion simulation and programming exercises by using the 3D model which use the external PLC and electrical lines to control the PC's internal structure.A8 software plays an important role in the communication, which make the virtual and the reality linked together.

\section{Building 3D-Models}

Because the controlled body is the mechanical model that we designed, the first step of the simulation is to design and draw the 3D model of the mechanism using the appropriate 3D modeling software.This paper takes tool change mechanism for machining center for example to describe the design process of mechanism's movement and simulation by Iria.

\section{Drawing and Designing 3D-Models}

Firstly, the mechanical structure of the simulation should be determined and the 2D procedure drawing should be designed.Then, the 3D-model of the mechanical structure in the drawings should be building[3].The three-dimensional modeling software the author uses is Catia.By designing,the $3 \mathrm{D}$-model of tool change mechanism for machining center has drawn in figure 2. 


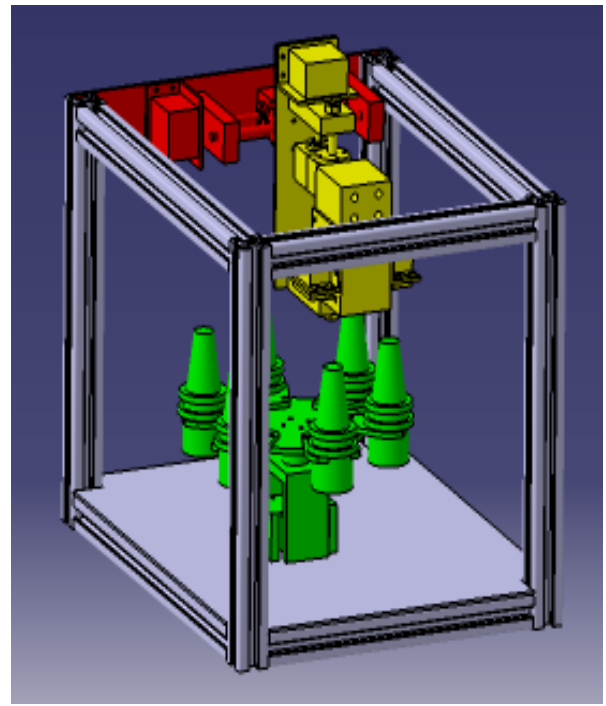

Figure 2.3D Model of Tool Change Mechanism for Machining Centers

\section{D-Models in Iria}

Irai software is the main platform for model simulation, the whole mechanical mechanism of threedimensional model is provided in this software platform for motion simulation.All motion simulations of the 3D-model of the whole mechanism are processing in the platform provided by this software.The first step is to import the $3 \mathrm{D}$-model we had previously drawn with Catia into Irai.The imported 3D-model of the tool change mechanism of the machining center is shown in Fig.3.

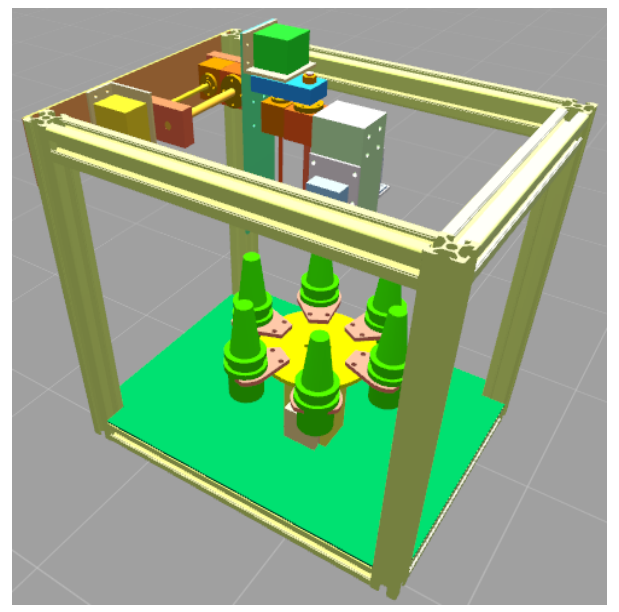

Figure 3.3D Model of Tool Change Mechanism for Machining Centers imported from Irai

When the 3D model just imports into Irai,all the constraints that set in the 3D modeling software all disappear[4].It is necessary to re-define the motion of each component in Irai so that all the parts can be recombined into a whole, in order to lay the foundation for the next step of motion simulation.

\section{Designing Intelligent 3D Resources and Systems}

Irai software can design new intelligent 3D resources and systems by re-applying 3D CAD models (computer-aided design). These 3D resources and systems can be saved independently or added to the Irai software repository for reuse in future 3D simulation model projects.In Irai, an intelligent 3D resource usually contains a number of action-attached 3D components (structured component assemblies), all of which, in the simulation process, constitute the intelligence of the resource.

In Irai, the relative position of the 3D model is defined by relative coordinates. Relatively refers to its matrix element, when the model is imported, each component has a default in the empty coordinate 
position under the local coordinate system (relative to the position of the base element).Movement or rotation can directly realized by the arrow or disk on the additional X, Y, Z coordinate system of each component of the imported 3D-model.If the size of the component need to be changed, it can be changed by modifying the dimension parameter in the component options.

Add actions to the $3 \mathrm{D}$ components and give 3D components intelligence.Adding motion to $3 \mathrm{D}$ components can build intelligent resources so that the models can move in Irai,interact with other $3 \mathrm{D}$ resources and communicate with each other or with the outside.For example,by adding sensors and actuators in the $\mathrm{X}, \mathrm{Y}, \mathrm{Z}$ rail of the $3 \mathrm{~d}$-model of tool change mechanism for machining center, the motion of the mechanism can be detected and feedback to users. The actions that add can be predefined and configurable, or they can be created by users through the integrated script editor.Their own custom actions are more complex than the software system's .Scripts are often used to simulate resources (internal logic) of the main controller, such as PLC. The movement relationship between the main body and the the components must be determined.Let one of the important parts of as a matrix [5]. The movement of other components are carried out around the substrate, in order to achieve the overall movement of the whole body[6]. The structure of the tree shows in figure 4 to help us to sort out the relationship between the various moving elements.

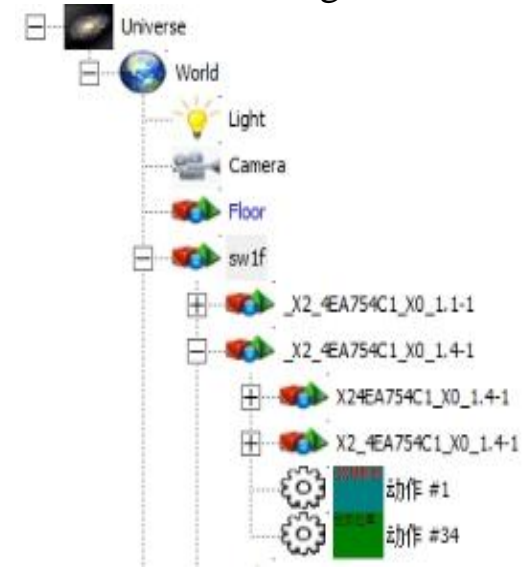

Figure 4.Motion Element Relationship Diagram

In the 3D-model of the tool change mechanism in the machining center, the required movement is the movement of the tool barrel along the $\mathrm{X}, \mathrm{Y}$ and $\mathrm{Z}$ directions[7]. The tool holder is rotated with the tool and rotates at a certain angle each time there is a corresponding action of the tool barrel to grab the tool and set the tool.These actions will eventually need to communicate with the actual PLC, through the preparation of PLC programs to control these movements. The specific parameters of the movement, such as movement speed, acceleration, displacement, etc must be determined before PLC program controls so as to ensure the definition of motion logic relations and various motion parameters in Irai consistent with the programming in the PLC.Irai can make the motion simulation more delicate based on our actual needs. The model is rendered by adjusting the physical engine performance in Irai.For example,the color of any component can be changed by the use of shaders in expert mode.It is also possible to show the effects of the fog or set the texture of the sky by adding artificial effects to the fog effect to increase its lifelike feeling.A human-computer interaction interface is established to realize the interaction between the operator and the 3D-model man-machine dialogue, and to better control the movement of the 3D-model.

\section{The Compilation of PLC Program}

When the definition of the 3D-model is complete,author should prepare a program for the external PLC, which must be interrelated with the actions that are defined in Irai and pay attention to the logic of the relationship between the various actions,

The list method can be used to analyze the order of the PLC program between the various actions to help us understand the analysis and reduce programming errors.PLC program requires the preparation of three stepper motor movement program, and three stepper motor movement has the 
order, and the movement time is also different, which needs to be consistent with the motion data of the components set in Irai.

\section{Communication between Irai Software and PLC}

Irai communicate with PLC by A8 which comes with Irai software[8].Author uses the Siemens S7200PLC as an example to introduce.First of all, the communication address of A8 and PLC must maintain the same.The motion port set in the 3D-model must be combined with the movement program of various components in the PLC set.Then add an outer join to the properties of the 3D model and select a connector (drive) in the external connection to implement a connection configuration with an external software / controller (PLC). Once the drive has been selected, the connection settings such as PLC IP address can be completed in the corresponding drive tab page.

After the communication is complete, we can carry on the simulation test to the designed structure through the simulation test function that Irai software has.

\section{Irai Software and PLC Simulation Experiment}

In order to meet the needs of mechatronics practice and in view of the current lack of PLC training equipment in vocational school and other issues,the virtual 3D-model of large-scale complex mechanical structure will be built in Irai,through the communication between the software and the external real PLC, which provide a platform for the experiment, and training of machine control ,PLC and $\mathrm{CNC}$ installation and maintenance and other mechatronics practice.Students control the virtual mechanical structure in computer to move by programming to the external PLC, in order to further deepen the understanding of the subject.Students can also design and develop the mechanical structure through the simulation platform, and use an external PLC to control it.This platform has a positive effect on improving students 'engineering practice ability and training teachers' scientific research and innovation ability.

\section{Conclusion}

The movement simulation experiment in the tool change mechanism of the machining center by Irai simulation software is researched. The original experimental method is optimized and the problems of less experimental equipment and risk in experiment are solved. At the same time, the applications of a kind of controller such as PLC is expanded.The dependence on the physical operating system is also reduced during the experiment, in line with the development direction of the times.

A good simulation platform for mechatronics has been built through Iria, which innovates the laboratory test methods. A series of problems due to complex mechanical structure, high cost and large space are solved.Students' interest in learning PLC software is improved and the interest in learning is also stimulated.Every student can do experiment simultaneously, which plays a very good experimental results and lay a solid foundation for the future use of such simulation platform.

\section{References}

[1] Liu jiguang,Yuan hao,Wang Ze,Wang yue.Design and Realization of Virtual Control Platform for Beer Production Line Based on Irai[J].Experimental Technology and Management,2015,32(6):123-127.

[2] Virtual Universe Pro V2 User Manual[M].Beijing.2013.

[3] LuoJun,Zhu Hongjie.Teaching Reform and Exploration of PLC Programmable Control Technology Course[J]. Western China Quality Education, 2016(10):37-39.

[4] Richter D. Infusing an interdisciplinary automation experience in engineering technology education[C]//Proceedings of the 2013 American Society for Engineering Education Annual Conference \& Exposition.Honolulu,2013. 
[5] Analog Devices. Inc. Data Sheet. CMOS 125 MHzcomplete DDS synthesizer AD9850[Z\}.2010,8(3):26-35.

[6] Gong Hanyue,Luo Qingsheng,Han Baoling,Research and Design of Virtual Control Platform for Tracked Robot[J].Computer Measurement \& Control,2012(3):694-696,701.

[7] Teng Yun.Analysis on Teaching Reform of PLC[J].Journal of Shandong Industry Technical College,2016(12):36-37.

[8] Han Yarong.The Reform of PLC Course Based on the Training Goal of Skilled Talents[J].China Training,2015(18):18-19. 